\title{
BASIC SCIENCE ARTICLE Repetitive noxious stimuli during early development affect acute and long-term mechanical sensitivity in rats
}

\author{
N. J. van den Hoogen $\mathbb{1}^{1,2}$, J. Patijn ${ }^{1}$, D. Tibboel ${ }^{3}$ and E. A. Joosten ${ }^{1,2}$
}

BACKGROUND: Prematurely born infants are frequently exposed to painful procedures in the neonatal intensive care unit, causing changes to the development of the nervous system lasting into adulthood. The current study aims to study acute and long-term consequences of neonatal repetitive noxious stimulation.

METHODS: Rat pups received either 4 or 10 unilateral needle pricks per day, while control littermates received 4 or 10 tactile stimuli in the first postnatal week. Behavioural sensitivity was assessed in the neonatal phase, in adulthood, and after re-injury of the same dermatome in adulthood.

RESULTS: An increase in the number of repetitive painful stimuli, from 4 to 10 needle pricks per day, resulted in increased mechanical hypersensitivity during the neonatal period. In adulthood, repetitive painful stimuli resulted in hyposensitivity to mechanical stimuli, while thermal sensitivity was unaffected. After re-injury of the same dermatome in adulthood, the number of repetitive noxious stimuli did not affect mechanical hypersensitivity. Both needle prick groups showed an increased duration of postoperative hypersensitivity compared to control.

CONCLUSION: This study shows that repetitive noxious stimulation during the early postnatal period affects acute and long-term mechanical sensitivity. Therefore, the amount of nociceptive stimuli should be minimized or adequately treated in a clinical setting.

Pediatric Research (2020) 87:26-31; https://doi.org/10.1038/s41390-019-0420-x

\section{INTRODUCTION}

Prematurely born infants are frequently exposed to painful procedures in the neonatal intensive care unit (NICU) and a subset is subsequently exposed to a variety of analgesic drugs. ${ }^{1,2}$ Clinical studies have shown that new-born neonates in the NICU undergo up to 14 painful procedures each day of admission, and this number has not decreased over the past 10 years. ${ }^{1-4}$ Several lines of evidence show that early life pain leads to changes in basal and post-surgical nociception in adulthood in both human studies and in experimental models, ${ }^{5-11}$ and persistent modalityspecific changes in sensory processing in extreme prematurely born infants who underwent procedural pain in early life were shown in an 11-year follow-up. ${ }^{8}$ Moreover, repeated exposure to invasive procedures places premature infants at particular risk, due to possible interference with the extensive developmental mechanisms and functional changes taking place in the central nervous system during the period in the NICU. ${ }^{12,13}$ The repetitive nature of small invasive noxious procedures experienced during NICU admittance will certainly affect the activity-dependent maturation of the nociceptive system in different ways than with a single stimulus. ${ }^{12}$ The amount of procedural pain in extremely preterm neonates was associated with somatosensory thalamic development previously. ${ }^{14}$ In addition, repeated invasive procedures will lead to prolonged exposure to noxiousness compared to a single noxious event. ${ }^{15}$ Until now, preclinical studies have modelled different procedures experienced during NICU admittance, like skin incision, inflammation, surgery, anaesthesia, maternal separation, and heel lances, showing long-term changes in sensitivity to mechanical, thermal, and re-injury stimuli. ${ }^{7,16-21}$ Repetitive needle pricking-induced pain in the first week following birth has shown to lead to alterations in spinal and supraspinal nociceptive processing at the neonatal phase, lasting into adulthood. ${ }^{16,17,22-24}$ Nevertheless, it remains unclear whether the number and intensity of pain experienced during early development is related to the severity of the long-term consequences. The current study aims to study the relation between the number of neonatal noxious events and the acute and long-term consequences hereof. Therefore, we have used a repetitive needle prick model as previously described. ${ }^{17,23}$ In this model, rat pups undergo 4 needle pricks into the hind-paw per day from day of birth (postnatal day 0 (P0)) to P7, leading to hypersensitivity during the neonatal phase. In addition, the repetitive needle prick model results in an increased duration of post-operative hypersensitivity in adulthood. ${ }^{17,23}$ By investigating the effect of different numbers of repetitive painful stimuli, ten instead of four, we aim to mimic the clinical situation more closely and assess how the number of repetitive painful stimuli during early development affects the maturation of the nociceptive system. Hence, we compared the effect of four versus ten needle pricks into the hind-paw on acute (neonatal) mechanical sensitivity, long-term adult mechanical and thermal sensitivity, and recovery of mechanical sensitivity after re-injury of the same dermatome in adulthood. We hypothesized that an increase in the number of needle pricks per day during the early development of the nociceptive system affects acute and long-term sensory sensitivity.

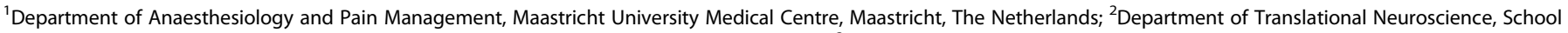

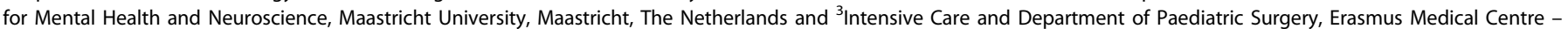
Sophia Children's Hospital, Rotterdam, The Netherlands

Correspondence: N. J. van den Hoogen (n.vandenhoogen@maastrichtuniversity.nl) 


\section{METHODS}

Animals

A total of 79 Sprague Dawley male and female rat pups were used. Rat pups (littermates) either received 4 or 10 tactile stimulations (cotton swab to the left hind-paw, 4 swabs $N=12$ males and 8 females; 10 swabs $N=8$ males and 12 females) or 4 or 10 noxious needle pricks ( 4 pricks $\mathrm{N}=9$ males and 11 females; 10 pricks $N=$ 10 males and 9 females). All rats were born on the 21 st or 22 nd day of gestation from time-pregnant Sprague-Dawley dams (Charles River, delivered at 13th day of gestation) at Maastricht University animal facility. Litters were culled to a maximum of ten pups. At P21, pups were weaned and housed in same-sex standard cages with 2-3 littermates in a temperature $\left(19-24^{\circ} \mathrm{C}\right)$ and humidity $(55 \pm 15 \%)$ controlled room with a reversed $12 \mathrm{~h} / 12 \mathrm{~h}$ day-night cycle. Food and water was available ad libitum for the duration of the study. All animals underwent behavioural testing in 3 blocks: neonatal (P0-P7), adult (P21-P56), and post-operative (P56-P65).

All animal experiments were performed in accordance with the European Directive for the Protection of Vertebrate Animals Used for Experimental and Other Scientific Purposes (86/609/EEC) and were approved by the Committee for Experiments on Animals, Maastricht, The Netherlands (DEC 2014-090).

Neonatal procedures and behaviour

To assess the effect of the amount of procedural pain, a neonatal repetitive needle prick model as described before was adapted and used. ${ }^{17,23}$ Rat pups received either 4 or 10 unilateral needle pricks per day from day of birth to P7 in the left hind paw, divided over a total of $4 \mathrm{~h}$ (4 times needle prick group, NP4, males $n=9$, females $n=11 ; 10$ times needle prick group, NP10, males $n=10$, females $n=9$ ). Tactile control pups were handled in the same way and received 4 or 10 tactile stimuli (4 times tactile control group, TC4, males $n=12$, females $n=8$; 10 times tactile control group, TC10, males $n=8$, females $n=12$ ) (Table 1). For each procedure, the entire nest was briefly separated from the dam and returned quickly. Mechanical sensitivity to von Frey filaments was assessed using dorsal application of calibrated Von Frey filaments (0.407; $0.692 ; 1.202 ; 2.041 ; 3.63$ (from P4 on); and 5.495 (from P6 on) before (baseline, BS) and 1, 3, and $5 \mathrm{~h}$ after the last needle prick each day (P0-P7). Each filament, starting with 0.407 , was applied 5 times to the dorsal surface of the hind-paws, until a 5 out of 5 paw withdrawal response was noted. After this, the measurement was completed and no higher filaments were applied. After the first block of behavioural testing, the animals were left with the dam until weaning to recover. At weaning, the injury to the left hindpaw was completely healed in all experimental groups, whereas no differences were observed in hind-paw condition in animals who underwent 4 or 10 needle pricks or who underwent tactile stimulation.

Mechanical sensitivity to Von Frey filaments

Mechanical sensitivity was assessed during development from weaning to adulthood (P21-P56, 3-8 weeks of age) using plantar application of calibrated Von Frey hairs as described previously. ${ }^{23}$ Briefly, animals were placed in a Plexiglas cage on a mesh floor.

\begin{tabular}{|ll|}
\hline Table 1. & Sample of animals for each experimental condition \\
\hline Condition & Animals \\
\hline TC4 & 12 males, 8 females \\
TC10 & 8 males, 12 females \\
NP4 & 9 males, 11 females \\
NP10 & 10 males, 9 females \\
\hline
\end{tabular}

Filaments with logarithmically increasing force were applied to the plantar surface of the hind-paws $(1.202 ; 2.041 ; 3.63 ; 5.495 ; 8.511$; 15.136; and $28.84 \mathrm{~g}$ ) for $5 \mathrm{~s}$ using the up-down method described by Chaplan. ${ }^{25}$ Mechanical sensitivity was tested each week from 3 to 8 weeks of age.

Thermal sensitivity to Hargreaves thermal test

Thermal sensitivity was assessed by measuring the paw withdrawal latency to a noxious thermal stimulus using a Hargreaves apparatus (Plantar test apparatus 37370, Ugo-Basille, Italy). Animals were placed in Plexiglas cages and a focus radiant heat source was placed under the ipsilateral hind-paw. A cut-off score of $20.1 \mathrm{~s}$ was implemented to prevent tissue damage.

Post-operative pain model in adulthood

At 8 weeks of age, all animals underwent a $1-\mathrm{cm}$ ipsilateral paw incision through the skin and fascia under isoflurane anaesthesia (4\% induction, 2-2.5\% maintenance). The underlying plantaris muscle was elevated using curved forceps and longitudinally incised over $1-2 \mathrm{~mm}^{26}$ Skin was sutured using 5-0 sutures, and animals were placed in their homecage for recovery. Mechanical sensitivity was tested as described above, 1, 3, 5, 7, and 9 days post-incision.

Data processing and analysis

From the Von Frey data, 50\% withdrawal thresholds were calculated and log transformation was performed to allow parametric testing. First, the effect of sex was assessed in all data sets. On neonatal, long-term, and perioperative behaviour, repeated-measures analysis of variance (ANOVA) with Bonferroni multiple testing corrections was performed (Graphpad Prism version 7.04). In addition, for the neonatal period an area under the curve (AUC) analysis was performed. For this, Graphpad Prism was used to calculate AUCs for all groups over the whole neonatal period (P0-P7). AUCs were compared using one-way ANOVA with Bonferroni multiple testing correction.

\section{RESULTS}

An increased number of neonatal repetitive needle prick stimulations increases ipsilateral mechanical sensitivity in the early postnatal period

Since there was no effect of sex on pain behaviour at any time point during the early postnatal period, data from male and female rat pups was pooled to increase power. Ipsilateral mechanical sensitivity was tested during the first week of life. Overall, mechanical threshold increased over time (effect of time: $F(31,2325)=32.02, p<0.0001)$. Mechanical sensitivity of needle prick animals increased over time, visible as a decrease in mechanical threshold compared to tactile control from P2 on. This effect was more pronounced in NP10 animals compared to NP4 animals (effect of procedure: $F(3,75)=3.746, p=0.0145$; interaction effect procedure and time: $F(93,2325)=1.921, p<$ 0.007). In addition, NP10 animals showed a deviation from tactile control more often compared to NP4 animals ( 21 versus 8 times). Subsequent AUC analysis of the entire neonatal period showed a significantly decreased AUC in NP10 animals compared to all other groups (effect of procedure: $F(3,75)=4.135, p<0.001$ ). The mechanical sensitivity of tactile control animals did not differ between TC4 and TC10 (Fig. 1).

An increased number of neonatal repetitive needle prick stimulations leads to ipsilateral mechanical hyposensitivity in adulthood but does not affect thermal sensitivity

Next, the effect of 4 or 10 neonatal repetitive procedures on ipsilateral mechanical and thermal sensitivity was assessed in developing rats, from P21 ( 3 weeks) to 8 weeks. Overall, mechanical sensitivity of all animals decreased over time, visible 


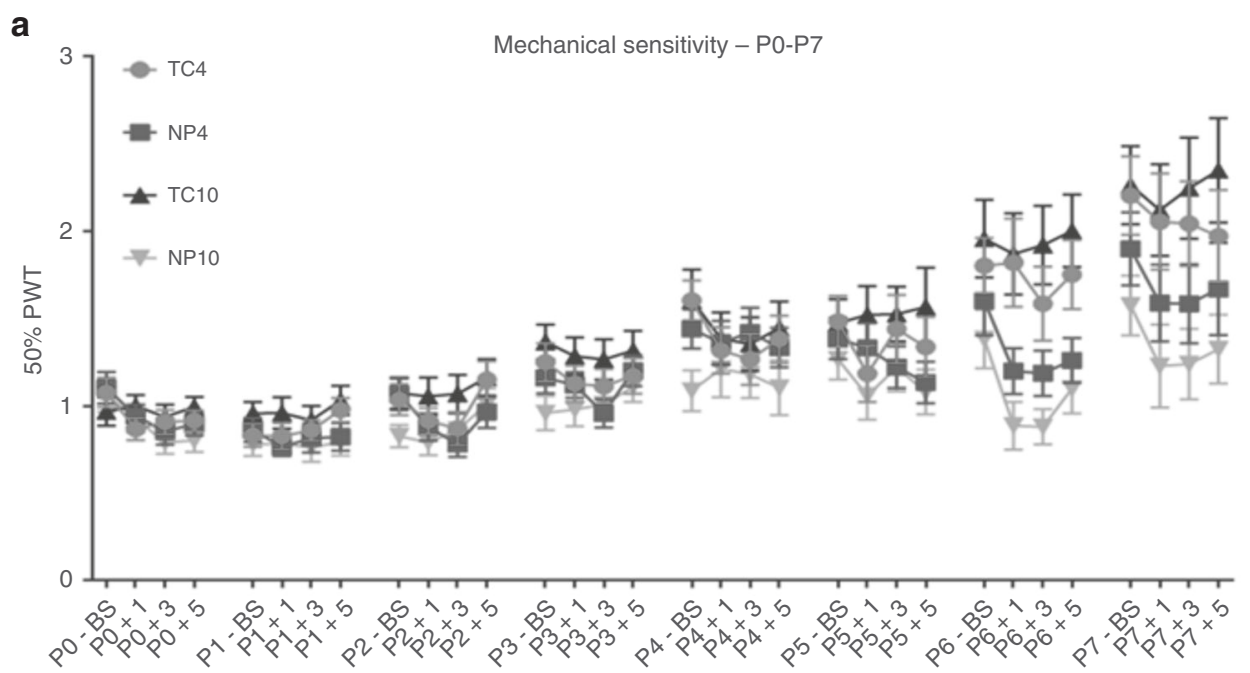

b
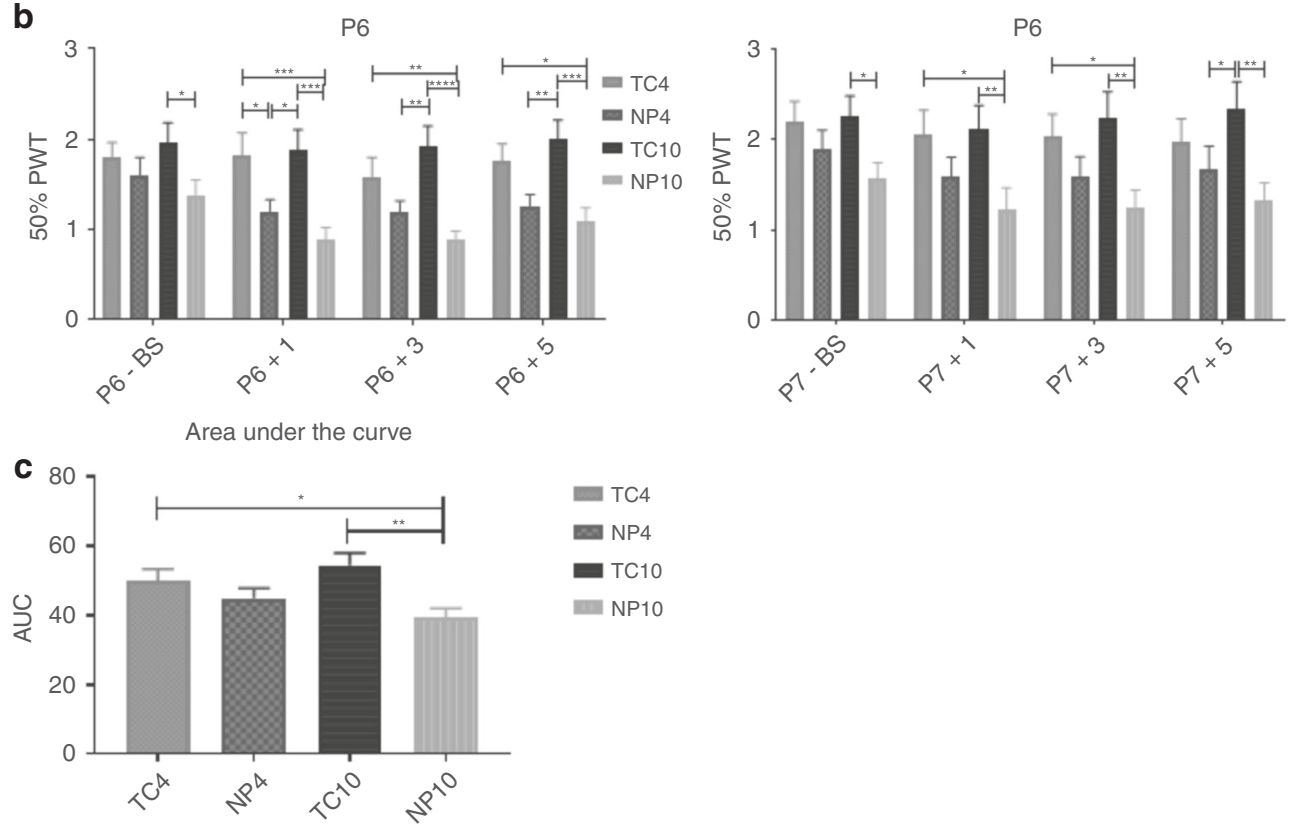

Fig. 1 Mechanical sensitivity during the first week of life following repeated neonatal painful procedures. Mechanical withdrawal thresholds in the ipsilateral paw at baseline (BS), and at 1,3 and $5 \mathrm{~h}$ after needle prick/tactile control (NP/TC). a Mechanical withdrawal threshold increased after the third postnatal day in tactile controls (TC4 and TC10, effect of time: $F(31,2325)=32.02, p<0.0001$, two-way analysis of variance (ANOVA)). Following needle pricks, mechanical withdrawal thresholds were significantly lower compared to tactile controls (effect of neonatal procedure: $F(3,75)=3.746, p=0.015$; interaction between procedure and time: $F(93,2325)=1.921, p<0.0001$, two-way ANOVA). The effect was more evident in animals receiving 10 needle pricks (NP10) compared to animals receiving 4 needle pricks (NP4). b shows the separate graphs for days P6 and P7. c Area under the curve (AUC) analysis over the whole neonatal period showed a significantly lower AUC in NP10 animals compared to other conditions (effect of procedure: $F(3,75)=4.135, p<0.001$ ). TC4: tactile control 4x; TC10: tactile control 10x; NP4: needle prick 4x; NP10: needle prick 10x. All data presented as mean \pm SEM. ${ }^{*} p<0.05,{ }^{* *} p<0.01$, ${ }^{* *} p<0.001$, ${ }^{* * *} p<0.0001$, between treatment comparisons

as an increase in $50 \%$ paw withdrawal threshold (effect of time: $F(4,300)=75.83, p<0.0001)$. Animals undergoing neonatal needle pricks (NP4 and NP10) showed significantly decreased mechanical sensitivity compared to tactile control animals (effect of neonatal procedure: $F(3,75)=3.901, p=0.012$ ). Post hoc analysis revealed no difference between NP4 and NP10 animals (Fig. 2).

In addition, thermal sensitivity was tested. There was no effect of time on thermal sensitivity (effect of time: $F(4,224)=0.9175, p$ $=0.4545)$. In addition, no differences were found at any time point between the neonatal procedure groups (effect of neonatal procedure: $F(2,56)=1.024, p=0.3658$ ) (Fig. 2).
An increased number of neonatal repetitive needle prick stimulations do not alter recovery of mechanical hypersensitivity after ipsilateral paw incision in adulthood

At the age of 8 weeks, animals from all groups underwent an ipsilateral paw incision as a model for acute post-surgical pain. All animals developed acute mechanical hypersensitivity from 1 day after incision (effect of time: $F(5,335)=93.96, p<0.0001$ ). Neonatal needle prick NP4 and NP10 significantly increased duration of post-incision mechanical hypersensitivity (effect of neonatal procedure: $F(2,67)=3.124, p=0.05$, no interaction effect). The mechanical sensitivity of both neonatal tactile control groups returned to pre-surgery baseline values 5 days after 

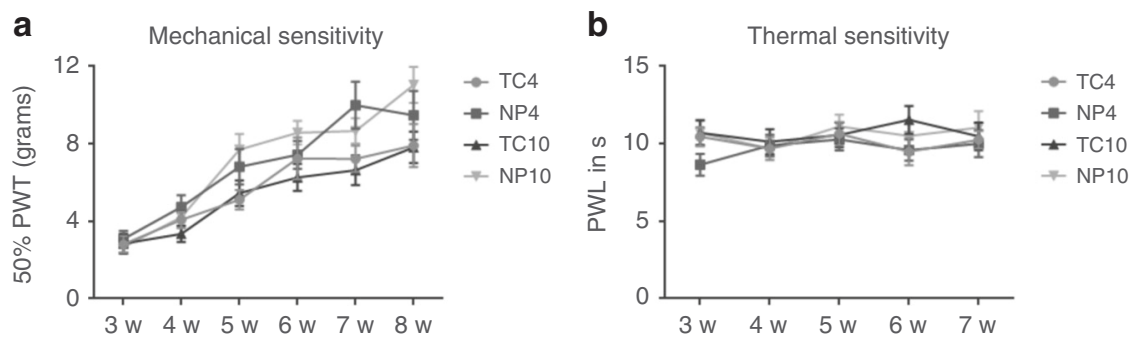

Fig. 2 Sensitivity to $\mathbf{a}$ mechanical and $\mathbf{b}$ thermal stimulation during development into adulthood following neonatal repeated procedures. a Mechanical withdrawal threshold increased over the weeks in all animals (effect of time: $p<0.0001$, two-way analysis of variance (ANOVA)), needle prick animals showed decreased mechanical sensitivity compared to tactile control (effect of neonatal procedure: $p=0.012$; interaction between procedure and time: $p=0.84$, two-way ANOVA). $\mathbf{b}$ Neither time nor neonatal procedures altered thermal sensitivity. Data presented as mean \pm SEM

incision, while both neonatal needle prick groups recovered at 7 days post-incision. Post hoc analysis revealed no difference in recovery between NP4 and NP10 animals (Fig. 3).

\section{DISCUSSION}

Behavioural analysis of mechanical sensitivity throughout development shows that the amount of nociceptive stimuli in early life affects both neonatal and adult sensitivity. More specifically, an increase in the number of repetitive painful stimuli, from 4 to 10 needle pricks per day from postnatal day P0 to P7, resulted in increased mechanical hypersensitivity during the neonatal period. From 3 to 8 weeks of age, repetitive painful stimuli in early life resulted in increased hyposensitivity to mechanical stimuli, while thermal sensitivity was unaffected. After re-injury of the same dermatome in adulthood, the number of repetitive noxious stimuli (4 compared to 10 needle pricks per day from P0 to P7) did not affect the intensity or duration of mechanical hypersensitivity. Both NP4 and NP10 showed an increased duration of postoperative hypersensitivity compared to animals undergoing tactile stimulation during early life, as shown previously for NP4. ${ }^{17,22,23}$

As shown before, this study showed acute mechanical hypersensitivity in animals undergoing needle pricks from P0 to P7. ${ }^{17,23}$ As hypothesized, we have shown that an increased number of four to ten repetitive noxious stimuli in the neonatal phase lead to increased acute hypersensitivity. In addition, we showed that increasing the number of tactile stimuli from four to ten per day did not alter acute mechanical sensitivity.

Next, the effect of an increased number of repetitive painful stimuli in the neonatal phase on long-term mechanical and thermal sensitivity in the adult was assessed. Thermal sensitivity, tested with a Hargreaves apparatus, was unaltered in the needle prick groups compared to tactile controls. However, this study shows a mechanical hyposensitivity from 5 to 8 weeks of age in animals undergoing 4 or 10 repetitive needle pricks per day from P0 to P7. The findings on hyposensitivity in adulthood after ten needle prick are in line with those reported using other, more invasive neonatal noxious stimulus models., 19,21,27 Models in which rats undergo tonic inflammatory pain during early life show thermal hyposensitivity after P34. ${ }^{21,28}$ In addition, surgical pain and anaesthesia lead to thermal and mechanical hyposensitivity in later life. ${ }^{7,27}$ It seems that a more severe pain stimulus in early life, whether tonic inflammation, post-surgical pain, or a large amount of needle pricks per day leads to hyposensitivity in adulthood in several animal models of neonatal pain. Our ten needle prick group might therefore resemble an inflammatory pain stimulus. Interestingly, we have found a hyposensitivity in animals undergoing four needle pricks during the first postnatal week as well. Previous studies by our group using this model did not show mechanical hyposensitivity in later life. ${ }^{17,23,29}$ However, several studies have suggested changes in nociception in adulthood after early life maternal separation, ${ }^{30,31}$ showing reduction in sensitivity in adult females after neonatal maternal separation. ${ }^{31}$ Possibly, the cumulative separation stress and needle pricks led to hyposensitivity as observed here. To fully understand this, a study using a separate group of maternal separated but not tactile or noxious stimulated animals would be necessary.

After re-injury to the same dermatome, animals undergoing repetitive neonatal needle pricks showed a longer duration of post-operative hypersensitivity to mechanical stimulation compared to tactile control animals. Interestingly, no differences in adult post-operative hypersensitivity were observed between animals undergoing 4 or 10 needle pricks per day from PO to P7. This suggests that the amount of neonatal needle pricks does not alter the intensity or duration of post-operative hypersensitivity after re-injury of the same dermatome, while it does alter baseline sensitivity to mechanical stimulation. It might be that the total plasticity caused by repetitive painful stimuli in early life is limited, and therefore an increase in repetitive noxious stimuli in early life is unable to increase the long-term effect on re-injury behaviour. Indeed, previous experiments showed a re-injury in adulthood following repetitive needle pricks in early life was not able to increase spinal excitability to the same extent as re-injury in naive animals. ${ }^{24}$ In order to further examine the plasticity capacity of the spinal nociceptive network in early life, more research using functional and molecular outcome measures is needed.

Overall, it seems that the stimulation-dependent physiological maturation of the nociceptive system is disturbed by early life overstimulation with noxious events. ${ }^{5,15,32-35}$ It is clear that the nociceptive physiological maturational processes are affected by nociceptive input in early life. This suggests that the immature nociceptive system of preterm infants admitted into the NICU is vulnerable to stress and painful stimuli. As maturation of the nociceptive system in early life is dependent on stimulation, overstimulation with noxious stimuli can have detrimental effects. As shown by this study, it seems that long-term pain behaviour is altered after a large, repetitive amount of needle pricks in early life. Possibly, treatment of early life pain would aid in preventing long-term changes to a developing nociceptive system, as shown before with paracetamol treatment in the $4 \times$ needle prick model. ${ }^{23}$

In conclusion, while an increased amount of painful input in the first week of life did not alter mechanical sensitivity after re-injury in adulthood, it does alter acute and adulthood sensitivity to mechanical threshold in a rat model of neonatal needle pricking. An increase of noxious stimuli further increased hypersensitivity during the neonatal period. These findings stress the need to reduce the number of painful procedures or to adequately treat procedural pain in this vulnerable age population. 

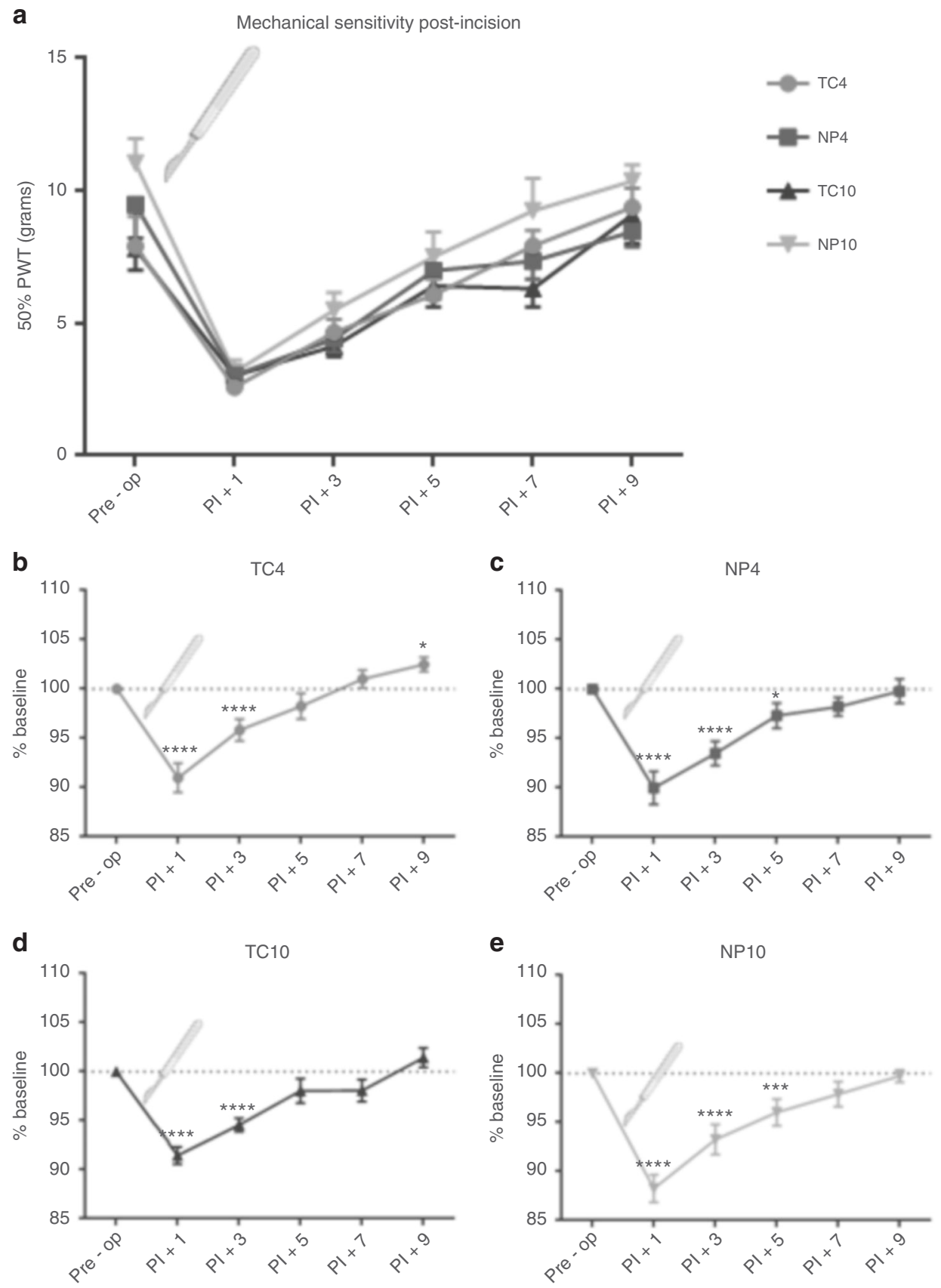

Fig. 3 Mechanical hypersensitivity after re-injury in adulthood following neonatal repeated procedures. Mechanical sensitivity was tested before (pre-op) and 1, 3, 5, 7, and 9 days (PI+1-PI+9) following incision. a shows the changes in mechanical withdrawal thresholds from all groups. Mechanical withdrawal threshold decreased in all animals following incision (effect of time: $F(5,335)=93.96, p<0.001$, two-way analysis of variance). b-e show the mechanical withdrawal thresholds in the ipsilateral paw per group normalized to baseline and presented as percentage of baseline. TC4 and TC10 animals showed a recovered mechanical sensitivity at 5 days post-incision, while NP4 and NP10 animals showed recovery 7 days post-incision. Data presented as mean $\pm \mathrm{SEM},{ }^{*} p<0.05$, ${ }^{* * *} p<0.001,{ }^{* * *} p<0.0001$, within treatment comparisons

\section{ACKNOWLEDGEMENTS}

This research was financially supported by the Pain Knowledge Centers from Maastricht and Rotterdam.

\section{ADDITIONAL INFORMATION}

Competing interests: The authors declare no competing interests.

Publisher's note: Springer Nature remains neutral with regard to jurisdictional claims in published maps and institutional affiliations.

\section{REFERENCES}

1. Simons, S. H. et al. Do we still hurt newborn babies? A prospective study of procedural pain and analgesia in neonates. Arch. Pediatr. Adolesc. Med. 157, 1058-1064 (2003).

2. Carbajal, R. et al. Sedation and analgesia practices in neonatal intensive care units (EUROPAIN): results from a prospective cohort study. Lancet Respir. Med. 3, 796-812 (2015).

3. Roofthooft, D. W., Simons, S. H., Anand, K. J., Tibboel, D. \& van Dijk, M. Eight years later, are we still hurting newborn infants? Neonatology 105, 218-226 (2014). 
4. Carbajal, R. et al. Epidemiology and treatment of painful procedures in neonates in intensive care units. JAMA 300, 60-70 (2008).

5. Walker, S. M. Neonatal pain. Paediatr. Anaesth. 24, 39-48 (2014).

6. Walker, S. M. Translational studies identify long-term impact of prior neonatal pain experience. Pain 158(Suppl 1), S29-S42 (2017).

7. Walker, S. M., Fitzgerald, M. \& Hathway, G. J. Surgical injury in the neonatal rat alters the adult pattern of descending modulation from the rostroventral medulla. Anesthesiology 122, 1391-1400 (2015).

8. Fitzgerald, M. \& Walker, S. M. Infant pain management: a developmental neurobiological approach. Nat. Clin. Pract. Neurol. 5, 35-50 (2009).

9. Brummelte, S. et al. Procedural pain and brain development in premature newborns. Ann. Neurol. 71, 385-396 (2012).

10. Grunau, R. E. et al. Neonatal pain, parenting stress and interaction, in relation to cognitive and motor development at 8 and 18 months in preterm infants. Pain 143, 138-146 (2009).

11. de Graaf, J. et al. Five-year follow-up of effects of neonatal intensive care and morphine infusion during mechanical ventilation on diurnal cortisol rhythm. J. Pediatr. 165, 459-63 e2 (2014).

12. Beggs, S., Torsney, C., Drew, L. J. \& Fitzgerald, M. The postnatal reorganization of primary afferent input and dorsal horn cell receptive fields in the rat spinal cord is an activity-dependent process. Eur. J. Neurosci. 16, 1249-1258 (2002).

13. Fitzgerald, M. \& Jennings, E. The postnatal development of spinal sensory processing. Proc. Natl. Acad. Sci. USA 96, 7719-7722 (1999).

14. Duerden, E. G. et al. Early procedural pain is associated with regionally-specific alterations in thalamic development in preterm neonates. J. Neurosci. 38 , 878-886 (2018).

15. Ranger, M. \& Grunau, R. E. Early repetitive pain in preterm infants in relation to the developing brain. Pain Manag. 4, 57-67 (2014)

16. Anand, K. J., Coskun, V., Thrivikraman, K. V., Nemeroff, C. B. \& Plotsky, P. M. Longterm behavioral effects of repetitive pain in neonatal rat pups. Physiol. Behav. 66, 627-637 (1999)

17. Knaepen, L. et al. Neonatal repetitive needle pricking: plasticity of the spinal nociceptive circuit and extended postoperative pain in later life. Dev. Neurobiol. 73, 85-97 (2013).

18. Ruda, M. A., Ling, Q. D., Hohmann, A. G., Peng, Y. B. \& Tachibana, T. Altered nociceptive neuronal circuits after neonatal peripheral inflammation. Science $\mathbf{2 8 9}$, 628-631 (2000).

19. Ren, K. et al. Characterization of basal and re-inflammation-associated long-term alteration in pain responsivity following short-lasting neonatal local inflammatory insult. Pain 110, 588-596 (2004).
20. Jennings, E. \& Fitzgerald, M. Postnatal changes in responses of rat dorsal horn cells to afferent stimulation: a fibre-induced sensitization. J. Physiol. 509(Pt 3), 859-868 (1998).

21. Laprairie, J. L. \& Murphy, A. Z. Neonatal injury alters adult pain sensitivity by increasing opioid tone in the periaqueductal gray. Front. Behav. Neurosci. 3, 31 (2009).

22. Chen, M. et al. Neonatal repetitive pain in rats leads to impaired spatial learning and dysregulated hypothalamic-pituitary-adrenal axis function in later life. Sci. Rep. 6, 39159 (2016).

23. van den Hoogen, N. J. et al. Neonatal paracetamol treatment reduces long-term nociceptive behaviour after neonatal procedural pain in rats. Eur. J. Pain 20, 1309-1318 (2016).

24. van den Hoogen, N. J. et al. Repeated touch and needle-prick stimulation in the neonatal period increases the baseline mechanical sensitivity and postinjury hypersensitivity of adult spinal sensory neurons. Pain 159, 1166-1175 (2018).

25. Chaplan, S. R., Bach, F. W., Pogrel, J. W., Chung, J. M. \& Yaksh, T. L. Quantitative assessment of tactile allodynia in the rat paw. J. Neurosci. Methods 53, 55-63 (1994).

26. Brennan, T. J., Vandermeulen, E. P. \& Gebhart, G. F. Characterization of a rat model of incisional pain. Pain 64, 493-501 (1996).

27. Sternberg, W. F., Scorr, L., Smith, L. D., Ridgway, C. G. \& Stout, M. Long-term effects of neonatal surgery on adulthood pain behavior. Pain 113, 347-353 (2005).

28. Ren, K., Novikova, S. I., He, F., Dubner, R. \& Lidow, M. S. Neonatal local noxious insult affects gene expression in the spinal dorsal horn of adult rats. Mol. Pain 1, 27 (2005).

29. van den Hoogen, N. J., van Reij, R. R., Patijn, J., Tibboel, D. \& Joosten, E. A. J. Adult spinal opioid receptor mu1 expression after incision is altered by early life repetitive tactile and noxious procedures in rats. Dev. Neurobiol. 78, 417-426 (2018).

30. Butkevich, I. P., Mikhailenko, V. A., Vershinina, E. A. \& Aloisi, A. M. Effects of neonatal pain, stress and their interrelation on pain sensitivity in later life in male rats. Chin. J. Physiol. 59, 225-231 (2016).

31. Weaver, S. A., Diorio, J. \& Meaney, M. J. Maternal separation leads to persistent reductions in pain sensitivity in female rats. J. Pain 8, 962-969 (2007).

32. Schwaller, F. \& Fitzgerald, M. The consequences of pain in early life: injury-induced plasticity in developing pain pathways. Eur. J. Neurosci. 39, 344-352 (2014).

33. Peters, J. W. et al. Does neonatal surgery lead to increased pain sensitivity in later childhood? Pain 114, 444-454 (2005).

34. Simons, S. H. \& Tibboel, D. Pain perception development and maturation. Semin. Fetal Neonatal Med. 11, 227-231 (2006).

35. Van den Hoogen, N. J., Patijn, J., Tibboel, D. \& Joosten, E. A. Neonatal plasticity of the nociceptive system: mechanisms, effects, and treatment of repetitive painful procedures during NICU admittance. Curr. Pharm. Des. 23, 5902-5910 (2017). 